\title{
Single-step fabrication of stressed waveguides with tubular depressed-cladding in phosphate glasses using ultrafast vortex laser beams
}

\author{
Guanghua Cheng Xuewen Long \\ State Key Laboratory of Transient Optics and Photonics, Xi'an Institute of Optics and Precision Mechanics, \\ Chinese Academy of Sciences, Xi'an 710119, China \\ e-mail address:gcheng@opt.ac.cn
}

\begin{abstract}
We report on the fabrication of the stressed optical waveguide with tubular depressedrefractive-index cladding in phosphate glasses by use of femtosecond vortex beam. Strained regions were emerged in domains surrounding the tubular track. Waveguiding occurs mainly within the tube induced by femtosecond laser.
\end{abstract}

\section{Introduction}

Ultrafast lasers emerged as promising tools to process refractive index changes in band-gap materials, resulting in waveguiding functions, has attracted a considerable interest for integrated 3D photonics since the pioneering work of Davis et al. [1]. However, in some materials including a large range of glasses, crystals and polymers, ultrafast laser exposure produces a negative refractive index variation due to matter rarefaction or volume damage in the laser induced tracks [2]. Therefore, light guidance within a single track becomes impossible. In order to fabricate waveguide to confine light in these materials effectively, pair line structure consisting of two parallel tracks on each side of the unexposed central domain was successfully demonstrated to confine light [3]. In this paper we demonstrate the fast single-step writing of stressed waveguides with tubular depressed-refractive-index cladding in phosphate glasses by the use of vortex laser pulse [4].

\section{Experiment}

A focused hollow beam can be directly generated by focusing vortex beams. The vortex beam can be created with a hologram employing a phase shift defined such as

$$
T_{n}(\theta)=\exp (\operatorname{in} \theta)
$$

where $\theta$ and $\mathrm{n}$ are polar angle and integer respectively. Therefore, a tubular structure can be fabricated by scanning the foci of the vortex fs laser beam parallel to the direction of laser propagation. Femtosecond laser pulses operating at a repetition rate of $1 \mathrm{kHz}$ with a center wavelength of $800 \mathrm{~nm}$ are generated by an amplified Ti: sapphire laser system (Spitfire, Spectra Physics). These pulses are reflected on a computer-controlled and electrically addressed spatial light modulator (SLM) (HEO1080P, HOLOEYE Photonics AG) for beam shaping. In order to obtain a higher beam quality we inserted in addition a phase term of a tilted plane wave to spatially separate and filter away the zero-order of diffraction which originates from the SLM itself. The laser pulses were focused using a $20 \times$ microscope objective.

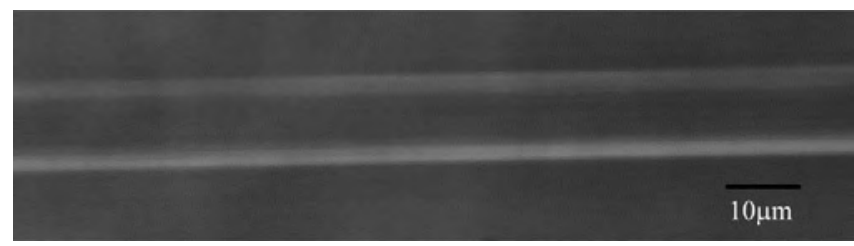

Fig. 1 PCM image of the waveguide with tubular depressed-refractive-index cladding (side view).

The ring-shaped intensity profile generated around the focal region was used to inscribe the tubular structures of material modification in a $4 \times 10 \times 10 \mathrm{~mm}^{3}$ polished phosphate glass parallelepiped using a longitudinal scheme. The sample was mounted on computer controlled high precision positioning stages and translated parallel with and towards the laser beam propagation direction at a rate of $10 \mu \mathrm{m} / \mathrm{s}$. For the fabrication of a high quality structure, the pulse energy is required not to exceed the threshold, which may otherwise result in beam collapse. In the experiment, the on-target pulse energy was set to $8 \mu \mathrm{J}$, and pulse duration was set to 600 fs. An Olympus BX51 positive phase contrast microscope (PCM) was employed to image the interaction region in a side-view geometry, 
showing relative variations of the refractive index translated into an intensity map. The image was recorded with a charge-coupled device (CCD) camera. Fig. 1 depicts the side-view PCM image of the tubular structure. The PCM image shows that the tubular track experienced a negative refractive index variation with respect to the pristine glass matrix (note white color in Fig. 1 indicative of negative index changes in positive PCM). The negative index change in exposed regions can most probably be related to the lower density as discussed below. Complementing the PCM information, the optical transmission microscope image of the exit plane of the obtained tubular structure is shown in Fig. 2(a), indicating the confinement of the laser modification. The diameter of the tubular track is $10 \mu \mathrm{m}$. In our exposure conditions, during photoinscription the deposition of the fs laser energy leads to a decrease of the local density and thus of the refractive index in the tubular track due to thermal expansion at the point of exposure of the laser beam [11]. The thermally-driven expansion, even for low repetition rates, causes compaction in the surrounding regions, which is responsible for an increase of the refractive index on axis, within the laser unexposed core. To check eventual stress-induced polarization effects, in Fig. 2(b) the tubular track under cross-polarization microscopy is shown. The polarizer was positioned underneath the sample stage with its pass axis fixed in the EastWest direction and the analyzer aligned with a pass axis oriented North-South. The intensity of the transmitted light through an optically-crossed polarizer and analyzer with a birefringent material in between is given by [5]

$$
\mathrm{I}(\varphi, \Delta \mathrm{n})=\mathrm{I}_{0} \sin ^{2}(2 \varphi) \sin ^{2}\left(\pi \Delta \mathrm{nd} / \lambda_{0}\right),
$$

where $\varphi$ is the angle of polarizer axis with the principal stress direction in the sample, $\Delta \mathrm{n}$ is the induced birefringence, $\mathrm{d}$ is the axial extension of the photo-modified region, and $\lambda_{0}$ is the wavelength of light used for imaging. Because principal stress direction is radial in the cross section of waveguide, a maximum brightness emerged when $\varphi=45,135,225,315^{\circ}$ in turn and the orientation of four bright petals keeps unchanged when the sample is rotated relative to the polarizer through $360^{\circ}$. Given the symmetry of compression, birefringence can not take place in the center of the core (under cross-polarization microscopy, extinction takes place in the center of the core shown in Fig. 2(b)). The increase of refractive index within the core of the tube due to stress-induced densification and depressed-refractive-index cladding support efficient light propagation. An attenuated $800 \mathrm{~nm} \mathrm{CW}$ laser radiation was coupled to one end facet of the waveguide by the aspheric lens mentioned above. The output emerging from the other end of the waveguide was imaged with a $20 \times$ microscope objective and recorded with a CCD camera. The near-field mode is about $12 \mu \mathrm{m}$ in diameter (at $1 / \mathrm{e}^{2}$ level), as shown in Fig. 2(c). A finite differences method for the reconstruction of refractive index profiles from near-field mode has been employed here to obtain the relative refractive index profile of the waveguide [12]. The maximum refractive index change is 0.004 , sufficient for single mode guiding.
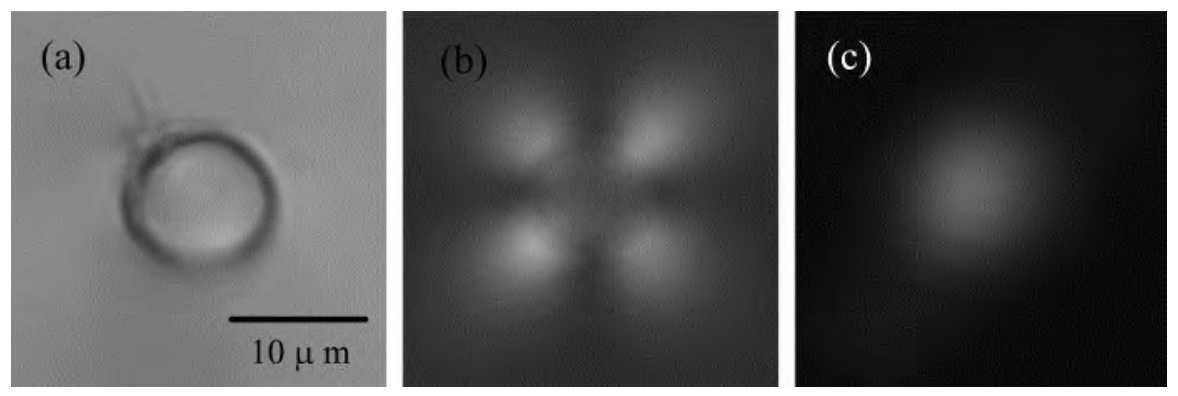

Fig. 2 (a) Optical transmission micrograph of the obtained tubular structure (end view). (b) Microscope image of the tubular track between crossed polarizers. (c) Near field mode of the tubular waveguide injected by $800 \mathrm{~nm}$ laser radiation. The field of view is the same. The scale bar is $10 \mu \mathrm{m}$

In summary, we introduced a method of single-step fabrication of stressed-induced optical waveguide with tubular depressed-refractive-index cladding in phosphate glasses by the use of focused femtosecond hollow laser beams.

\section{References}

[1] K. M. Davis, K. Miura, N. Sugimoto, and K. Hirao, "Writing waveguides in glass with a femtosecond laser," Opt. Lett. 21, 1729-1731 (1996). [2] J. W. Chan, T. R. Huser, S. H. Risbud, J. S. Hayden, and D. M. Krol, "Waveguide fabrication in phosphate glasses using femtosecond laser pulses, ” Appl. Phys. Lett. 82, 2371-2373 (2003).

[3] W. F. Silva, C. Jacinto, A. Benayas, J. R. Vazquez de Aldana, G. A. Torchia, F. Chen, Y. Tan, and D. Jaque, "Femtosecond-laser-written, stress-induced Nd:YVO4 waveguides preserving fluorescence and Raman gain,” Opt. Lett. 35, 916-918 (2010).

[4]Xuewen Long, Jing Bai, Wei Zhao, Razvan Stoian, Rongqing Hui, and Guanghua Cheng, "Stressed waveguides with tubular depressedcladding inscribed in phosphate glasses by femtosecond hollow laser beams," Opt. Lett. 37, 3138-3140 (2012) 